\title{
Simultaneous LQ Optimal Control Design for Discrete-time Systems and Sampled-data Systems ${ }^{1}$
}

\author{
Yong-Yan Cao ${ }^{\dagger} \quad J_{a m e s} L^{\ddagger} \quad$ You-Xian Sun ${ }^{\xi}$ \\ ${ }^{\dagger}$ Dept. of Elec. Eng., University of Virginia, Charlottesville, VA 22903, USA \\ email: yycao@virginia.edu \\ ${ }^{\ddagger}$ Dept. of Mech. Eng., The University of Hongkong, Pokfulam, Hong Kong \\ ${ }^{\xi}$ Inst. of Industrial Process Control, Zhejiang University, Hangzhou 310027, China
}

\begin{abstract}
This paper is concerned with simultaneous LQ optimal control design for discrete-time and sampleddata systems. First it is shown that discrete-time simultaneous LQ design problem of a set of discrete-time systems can be reduced to solving a set of coupled matrix inequalities. An iterative LMI algorithm is presented to solve the feasibility and the feedback gain. Then simultaneous stabilization and simultaneous LQ optimal control of a set of LTI continuous-time systems is considered via piecewise constant output feedback. It is shown that the design of a periodic piecewise constant feedback gain simultaneously minimizing a set of given continuous-time performance indexes for a set of LTI continuous-time systems can be reduced to that of a constant feedback gain minimizing a set of equivalent discrete-time performance indexes for a set of LTI discrete-time systems. Explicit algorithms for computing the equivalent discrete-time systems and performance indexes are derived. Examples are used to demonstrate the effectiveness of the proposed method.
\end{abstract}

Key Words: simultaneous stabilization, optimal control, periodic systems, piecewise constant output feedback (COF).

\section{Introduction}

Recently, an iterative linear matrix inequality approach was proposed to obtain output feedback and state feedback gain for a collection of continuous-time (CT) plants in [3]. Cao et al. [4] proved that a finite number of CT plants are simultaneously stabilizable via state feedback or static output feedback if and only if a set of coupled LQ control problems with some compatible cross terms in the cost functional is feasible and then it is reduced to a coupled algebraic Riccati inequalities (ARIs) problem. An iterative LMI (ILMI) algorithm is presented to obtain a feasible solution. Lam and Cao [10] addressed the linear-quadratic simultaneous optimal control design via static output feedback and state feedback using iterative

\footnotetext{
${ }^{1}$ This work was partially supported by the Alexander von Humboldt foundation and the HKU CRCG Grant No.10203013.
}

LMI approach for a set of CT systems.

Recent work on linear time-varying control has demonstrated that simultaneous stability can be easily carried out by using a time-varying controller [8]. A multirate approach is presented in Khargonekar et al. [8], where a simultaneously stabilizing control law based on periodic dynamic compensators is proposed. The basic idea is to divide an output sampling interval into as many subintervals as there are plants to be stabilized and to implement a deadbeat controller for each plant. This problem is also studied in [7]. Although the approach employed is quite general, an issue that it does not address is the closed-loop performance. This is because excessive intersampling ripple may occur under the restriction of deadbeat control. As a consequence, the closed-loop system may exhibit large overshoot and sensitive to parameters in many cases. Here we measure the performance in an LQR sense: for each possible model we choose a standard LQR-type cost function, and then we show how to design a piecewise constant controller which not only simultaneously stabilizes each model but also minimizes the sum of the performance indexes.

In this paper, simultaneous optimal control design is first addressed using LMI technique for a set of discretetime (DT) plants. We show that the design of simultaneous stabilization optimal controller for a set DT plants can be reduced to an optimization problem subject to a set of coupled matrix inequalities. The guaranteed-cost performance design can also be treated using the ILMI algorithm. Simultaneous optimal design of a set of linear time-invariant systems using periodic piecewise COF is also considered. We show that the problem can be reduced to the design of a COF gain minimizing a set of equivalent DT performance indexes for a set of LTI DT systems. Explicit algorithms for computing the equivalent DT systems and performance indexes are obtained.

\section{Simultaneous LQ Optimal Control for DT Systems}

Consider the following $r$ DT systems:

$$
x_{i}(k+1)=A_{i} x_{i}(k)+B_{i} u_{i}(k), y_{i}(k)=C_{i} x_{i}(k),
$$

where $x_{i} \in \mathcal{R}^{n_{i}}$ is the state, $u_{i} \in \mathcal{R}^{m}$ the control, $y_{i} \in \mathcal{R}^{p}$ 
the output for $i=1, \ldots, r . A_{i}, B_{i}$, and $C_{i}$ in (1) are constant and with appropriate dimensions. We assume that the triple $\left(A_{i}, B_{i}, C_{i}\right)$ is stabilizable and detectable. The problem considered here is the design of a static output feedback control law

$$
u_{i}(k)=F y_{i}(k)
$$

which minimizes an upper bound on the performance measures

$$
J_{i}=\mathcal{E}\left\{\sum_{k=0}^{\infty}\left[\begin{array}{l}
x_{i}(k) \\
u_{i}(k)
\end{array}\right]^{T}\left[\begin{array}{ll}
Q_{i} & S_{i} \\
S_{i}^{T} & R_{i}
\end{array}\right]\left[\begin{array}{l}
x_{i}(k) \\
u_{i}(k)
\end{array}\right]\right\},
$$

for $i=1, \ldots, r$, where $Q_{i} \geq 0, R_{i}>0$ and $Q_{i}$ $S_{i} R_{i}^{-1} S_{i}^{T} \geq 0$. In (3), the expected values are used to take into account the dependence of $J_{i}$ on the initial conditions. We shall assume that $x_{i}(0)$ is a random variable with zero mean and unit covariance, i.e. it satisfies $\mathcal{E}\left[x_{i}(t)\right]=0$ and $\mathcal{E}\left[x_{i}(0) x_{i}^{T}(0)\right]=I$. We assume that all systems have $\left(A_{i}, B_{i}\right)$ controllable and $\left(A_{i}, Q_{i}^{1 / 2}\right)$ observable. It is well known that the performance measures in (3) are given by

$$
J_{i}=\operatorname{trace}\left(P_{i}\right) \text {, }
$$

where each $P_{i} \geq 0$ satisfies, for a given $F$, the Lyapunov equation

$$
\hat{A}_{i}^{T} P_{i} \hat{A}_{i}+\bar{Q}_{i}+\left(F C_{i}\right)^{T} R_{i} F C_{i}=P_{i}
$$

for $i=1, \ldots, r$, where $\hat{A}_{i}=A_{i}+B_{i} F C_{i}, \bar{Q}_{i}=Q_{i}+$ $S_{i} F C_{i}+\left(S_{i} F C_{i}\right)^{T}$.

Theorem 1 Consider systems (1) with weighting matrices $\left(Q_{i}, R_{i}, S_{i}\right)$ for $i=1, \ldots, r$. The ith system is stabilizable via static output feedback if and only if there exist matrices $F$ and $G_{p i}$ such that

$$
\hat{R}_{i}^{1 / 2} F C_{i}+\hat{R}_{i}^{-1 / 2}\left(B_{i}^{T} P_{i} A_{i}+S_{i}^{T}\right)=G_{p i},
$$

where $\hat{R}_{i}=R_{i}+B_{i}^{T} P_{i} B_{i}, P_{i} \geq 0$ is the solution of the algebraic Riccati equation

$$
\begin{array}{r}
A_{i}^{T} P_{i} A_{i}-\left(A_{i}^{T} P_{i} B_{i}+S_{i}\right) \hat{R}_{i}^{-1}\left(B_{i}^{T} P_{i} A_{i}+S_{i}^{T}\right) \\
+Q_{i}+G_{p i}^{T} G_{p i}=P_{i} .
\end{array}
$$

In this case, the performance index takes the value $J_{i}=$ trace $\left(P_{i}\right)$. If there exist real matrices $F$ and $G_{p i}$ such that the Riccati equations (6) have solutions $P_{i} \geq 0$ for $i=1, \ldots, r$, then the feedback gain $F$ simultaneously stabilizing the $r$ systems and minimizing the performance indexes (3) for $i=1, \ldots, r$.

Theorem 1 gives a necessary and sufficient condition for static output feedback stabilizability for a single DT system. When $G_{p i}=0,(6)$ is the standard Riccati equation to construct state feedback control law minimizing the performance index (3). On the other hand, if Riccati equation (6) has a solution $P_{i} \geq 0$ with $G_{p i}=0$ and $F$ can be selected to satisfy constraint (5), from optimal control theory it is the optimal feedback gain via state feedback. Theorem 1 is an extension of the result of [9] for DT systems, where a similar necessary and sufficient condition on the static output feedback stabilizability was established for the CT systems with $Q_{i}=C_{i}^{T} C_{i}, S_{i}=0$ and $R_{i}=I$. Since the concepts such as stabilizability and detectability are not explicitly employed in Theorem 1, the proof is much more transparent. Note that this result is also presented in [6].

For a feedback gain $F$, which simultaneously stabilizes the $r$ systems, it generally does not minimize every performance index in (3) of the corresponding single plant, but there exist $P_{i} \geq 0$ such that the following Lyapunov inequalities hold

$$
\hat{A}_{i}^{T} P_{i} \hat{A}_{i}+\bar{Q}_{i}+\left(F C_{i}\right)^{T} R_{i} F C_{i}<P_{i} .
$$

In general, the performance values $\operatorname{trace}\left(P_{i}\right) \geq \operatorname{trace}\left(P_{i}^{0}\right)$ for $i=1, \ldots, r$, where $P_{i}^{0} \geq 0$ is the solution of Lyapunov equation (4). Therefore, we shall determine an output feedback gain to minimize the sum of the performance index (3), that is,

$$
J=\sum_{i=1}^{r} \operatorname{trace}\left(P_{i}\right)
$$

where $P_{i} \geq 0$ satisfying (7) for $i=1, \ldots, r$.

In practical control design, it is always desirable to design a control system which is not only stable, but also guarantees an adequate level of performance for every possible operating condition. In this paper, the guaranteedcost performance design will also be treated and a minimization of a bound on all the performance measures, $\gamma$, is sought such that

$$
\operatorname{trace}\left(P_{i}\right) \leq \gamma, \quad i=1, \ldots, r
$$

\section{ILMI Approach to Simultaneous Op- timal Design}

Note that (7) is equivalent to

$$
\left[\begin{array}{cc}
\bar{Q}_{i}-P_{i} & A_{i}^{T} P_{i} \\
P_{i} A_{i} & -P_{i}-P_{i} B_{i} R_{i}^{-1} B_{i}^{T} P_{i}
\end{array}\right]+\Gamma_{i}^{T} \Gamma_{i}<0
$$

where $\Gamma_{i}=\left[\begin{array}{ll}R_{i}^{1 / 2} F C_{i} & R_{i}^{-1 / 2} B_{i}^{T} P_{i}\end{array}\right]$, which is a quadratic matrix inequality. As a result of the negative sign in the $-P_{i} B_{i} R_{i}^{-1} B_{i}^{T} P_{i}$ term, (10) cannot be simplified to an LMI. To accommodate the $-P_{i} B_{i} R_{i}^{-1} B_{i}^{T} P_{i}$ term, we introduce an additional design variable $X_{i}$. Note that

$$
\left(X_{i}-P_{i}\right)^{T} B_{i} R_{i}^{-1} B_{i}^{T}\left(X_{i}-P_{i}\right) \geq 0 .
$$

Therefore, we obtain a sufficient condition for the feasibility of the inequality (10), given by

$$
\left[\begin{array}{cc}
\widetilde{Q}_{i}-P_{i} & A_{i}^{T} P_{i} \\
P_{i} A_{i} & -P_{i}-\Theta\left(P_{i}, X_{i}\right)
\end{array}\right]+\Gamma_{i}^{T} \Gamma_{i}<0,
$$


where $\Theta\left(P_{i}, X_{i}\right)=X_{i} B_{i} R_{i}^{-1} B_{i}^{T} P_{i}+P_{i} B_{i} R_{i}^{-1} B_{i}^{T} X_{i}$ $X_{i} B_{i} R_{i}^{-1} B_{i}^{T} X_{i}$. The following theorem can be established along a similar line as in [10].

Theorem 2 There exists a solution $\left(P_{i}>0, F\right)$ satisfying matrix inequality (10) if and only if there exist matrices $F, P_{i}>0$ and $X_{i}>0$ satisfying matrix inequality (11) for $i=1, \ldots, r$.

Using the Schur complement, inequality (11) is equivalent to the following matrix inequality

$$
\left[\begin{array}{ccc}
\bar{Q}_{i}-P_{i} & A_{i}^{T} P_{i} & * \\
P_{i} A_{i} & -P_{i}-\Theta\left(P_{i}, X_{i}\right) & * \\
R_{i}^{1 / 2} F C_{i} & R_{i}^{-1 / 2} B_{i}^{T} P_{i} & -I
\end{array}\right]<0
$$

This matrix inequality points to an iterative approach to solve for $F$ and $P_{i}$, namely, if $X_{i}$ is fixed in (12), then it reduces to an LMI problem on the unknown $F$ and $P_{i}$. The LMI problem is convex and can be solved if a feasible solution exists.

When $X_{i}$ is fixed, however, LMI (12) is only a sufficient condition for the feasibility of (10). In fact, if we find a solution of LMI (12), then we find a solution of (10). But, in general, it has no solution for a fixed $X_{i}$. On the other hand, we can solve the optimal output feedback problem repeatedly for modified systems with the pair $\left(A_{i}, B_{i}, C_{i}\right)$ replaced by scaled pairs of the form $\left(A_{i} / \beta^{j}, B_{i} / \beta^{j}, C_{i}\right)$, where $\beta^{j} \geq 1$. This means that we can first determine a solution $F^{j}$ such that $\rho\left(A_{i}+\right.$ $\left.B_{i} F^{j} C_{i}\right)<\beta^{j}$, that is, the closed-loop system matrices $A_{i}+B_{i} F^{j} C_{i}$ have eigenvalues in the $\beta^{j}$-circle of in the complex $s$-plane. From the above derivation we can obtain a necessary condition for the feasibility of (10), i.e., (10) has a solution $\left(P_{i}>0, F\right)$ then there exist a real number $\beta \geq 1$ and a matrix $X_{i}>0$ such that

$$
\left[\begin{array}{ccc}
\bar{Q}_{i}-P_{i} & A_{i}^{T} P_{i} & * \\
P_{i} A_{i} & -\beta^{2} P_{i}-P_{i} B_{i} R_{i}^{-1} B_{i}^{T} P_{i} & * \\
R_{i}^{1 / 2} F C_{i} & R_{i}^{-1 / 2} B_{i}^{T} P_{i} & -I
\end{array}\right]<0 .
$$

Based on the idea that all eigenvalues of $A_{i}+B_{i} F C_{i}$ are reduced progressively towards the unit circle through the reduction of $\beta$, we may close in on the feasibility of $(10)$. In other words, a stabilizing output gain will be found once $\beta \leq 1$. This technique can even be used to achieve a prescribed stability degree for the closed-loop system.

The following algorithm is an extension of the algorithm proposed in [10].

\section{ILMI Algorithm for DT systems}

Step 1. SET $j=1$, and SOLVE the following DT algebraic Riccati equation for

$P_{i}=A_{i}^{T} P_{i} A_{i}-\left(A_{i}^{T} P_{i} B_{i}+S_{i}\right) \hat{R}_{i}^{-1}\left(B_{i}^{T} P_{i} A_{i}+S_{i}^{T}\right)+Q_{i}$,

SET $X_{i}^{j}=P_{i}$ as the initial guess of the solution of (11), for $i=1, \ldots, r$.
Step 2. SOLVE the following optimization problem for $P_{i}^{j}, F^{j}$, and $\beta^{j}$.

OP1: Minimize $\beta^{j}$ subject to the following LMI constraints:

$$
\left[\begin{array}{ccc}
\bar{Q}_{i}-P_{i}^{j} & A_{i}^{T} P_{i}^{j} & * \\
P_{i}^{j} A_{i} & -\left(\beta^{j}\right)^{2} P_{i}^{j}-\Theta\left(P_{i}^{j}, X_{i}^{j}\right) & * \\
R_{i}^{1 / 2} F C_{i} & R_{i}^{-1 / 2} B_{i}^{T} P_{i}^{j} & -I
\end{array}\right]<0,
$$

Denote $\hat{\beta}^{j}$ as the minimized value of $\beta^{j}$.

Step 3. IF $\hat{\beta}^{j} \leq 1, \operatorname{SET} \beta^{j}=1$, ELSE SET $\beta^{j}=\hat{\beta}^{j}$.

Step 4. SOLVE the following optimization problem for $P_{i}^{j}$ and $F^{j}$.

OP2: Minimize $\sum_{i=1}^{r} \operatorname{trace}\left(P_{i}^{j}\right)$ subject to the above LMI constraints (14) and (15).

Denote $\hat{P}_{i}^{j}$ and $\hat{F}^{j}$ as respectively the solutions $P_{i}^{j}$ and $F^{j}$ of OP2.

Step 5. IF $\sum_{i=1}^{r}\left\|\operatorname{trace}\left(X_{i}^{j}-\hat{P}_{i}^{j}\right)\right\|<\delta$, a pre-determined tolerance, GOTO Step 6, ELSE GOTO Step 7.

Step 6. IF $\beta^{j}=1$, obtain the optimal solution $P_{i}^{\text {opt }}=$ $\hat{P}_{i}^{j}, F^{\text {opt }}=\hat{F}^{j}$. STOP

ELSE this algorithm cannot get a feasible solution. STOP

Step 7. SET $j=j+1$ and $X_{i}^{j}=\hat{P}_{i}^{j-1}$. IF $\beta^{j-1}=1$, GOTO Step 4, ELSE GOTO Step 2.

For the guaranteed-cost design, only the linear inequality constraints trace $\left(P_{i}^{j}\right) \leq \gamma, \quad i=1, \cdots, r$ are required to be added in OP1 and OP2. When compared with the previous approaches, the present technique uses $F$ as a free design variable in the LMI optimization framework. Moreover, with $F=\left[f_{i j}\right]_{m \times p}$, the controller gain can be constrained element-by-element in the following form

$$
\varphi_{i j}<f_{i j}<\psi_{i j}, \quad i=1, \ldots, m, j=1, \ldots, p .
$$

These constraints are linear and hence can also be incorporated naturally in the optimization process.

The above algorithm can also be used to compute a decentralized feedback gain, i.e., the gain with zero nondiagonal blocks. The state feedback optimal design corresponds to the special case where $C_{i}=I$. With $Q_{i}=0$ and $R_{i}=I$, the proposed algorithm then determines the simultaneous stabilizability of $r$ DT plants via static output feedback.

\section{Simultaneous LQ Design for Multirate SD Systems}

To make a distinction, CT signals will be represented by (.) around an independent variable, whereas DT signals will be represented by bracket $[\cdot]$ in the following. 
It is interesting to explore the possibility to simultaneously stabilize a collection of linear time-invariant systems using periodic time-varying output feedback gains. A trivial necessary condition that the optimal multi-model constant/static output feedback problem has a solution for a time-invariant multi-model system of the form (1) is that there exists a stabilizing output feedback gain which simultaneously stabilizes all the systems. It is well known that not all systems are stabilizable by static output feedback thus the approach proposed in above section is not generally applicable. However, analogous to the single model case we can try to employ periodic static output feedback for the control of linear time-invariant systems which are not stabilizable via static output feedback. From $[1,5,8]$, it is known that provided the period $N$ is chosen sufficiently high every time-invariant system can be stabilized by using periodic COF. Moreover, not only can each model of the multi-model system be stabilized by a periodic COF but an arbitrary fast dynamics can also be achieved simultaneously for all the models. The increased stabilization potential of the periodic output feedback applied to multi-models has been shown in $[2,7,8]$. Thus, periodic static output feedback control could be a very promising approach for practical multimodel applications.

Consider the following LTI CT systems

$$
\dot{x}_{i}(t)=A_{i} x_{i}(t)+B_{i} u(t), y_{i}(t)=C_{i} x_{i}(t),
$$

where $i=1, \ldots, r$. The objective is to design, if possible, a controller which performs well for each of these models. Assume that the sampling period is $h_{0}$, where $h_{0}$ is to be chosen such that $\left(e^{A_{i} h_{0}}, C_{i}\right)$ is detectable for $i=1, \ldots, r$. The output measurements are taken at time instants $t=$ $k h_{0}, k=0,1,2, \cdots$. The periodic output feedback control law is given by

$$
u(t)=F(t) y\left(k h_{0}\right), \text { for } k h_{0} \leq t<(k+1) h_{0},
$$

where $F\left(t+h_{0}\right)=F(t)$, which simultaneously minimizes the following CT performance index

$$
J_{i}=\mathcal{E}\left\{\int_{0}^{\infty}\left[\begin{array}{l}
x_{i}(t) \\
u_{i}(t)
\end{array}\right]^{T}\left[\begin{array}{ll}
Q_{i} & 0 \\
0 & R_{i}
\end{array}\right]\left[\begin{array}{l}
x_{i}(t) \\
u_{i}(t)
\end{array}\right] d t\right\}
$$

for $i=1, \ldots, r$.

We consider a controller which samples all the plant outputs with the same period $h_{0}$, and changes the $i$-th plant input with shorter period $h_{i}$ (and keeps it constant over the time interval of $h_{i}$ ), where $h_{i}=h_{0} / N_{i}$, and $N_{i}$ are some positive integers. $h_{0}$ is called frame period, and $N_{i}$ input multiplicity. This means that each component is fed into the system by means of the following zero-order hold mechanism

$$
u_{i}(t)=u_{i}\left(j h_{i}\right), \quad t \in\left[j h_{i},(j+1) h_{i}\right)
$$

As in $[1,7]$, the control law is assumed to be piecewise constant, i.e. it is given by

$$
u_{i}\left(k h_{0}+j h_{i}\right)=f_{i j}(k) y\left(k h_{0}\right)
$$

where $f_{i j}(k)$ is a row vector denoting the time-varying feedback gain for $i=1, \ldots, m, j=0, \ldots, N_{i}-1$. This type of controller is called multirate input COF controller. Let $N$ be the least-common multiple of the entries of $\left\{N_{i} \mid i=1, \ldots, m\right\}$ and $h=h_{0} / N, n_{i}=N / N_{i}$. In the following, we will also denote

$$
\begin{aligned}
u[k N+j] & =F_{j}(k) y[k N], \\
F_{j}(k) & =\left[\begin{array}{lll}
f_{1 j_{1}}(k)^{T} & \cdots & f_{m j_{m}}(k)^{T}
\end{array}\right]^{T},
\end{aligned}
$$

for $j=0, \ldots, N-1, j_{i}=0, \ldots, N_{i}-1$. For simplicity, we first consider the following single CT system

$$
\dot{x}(t)=A x(t)+B u(t), y(t)=C x(t) .
$$

It is assumed that $(A, B)$ and $(A, C)$ are assumed to be controllable and observable, respectively. Let $\left(A_{d}, B_{d}, C\right)$ denote the DT system of $(22)$ at rate $1 / h$, i.e., $A_{d}=$ $\exp (A h), B_{d}=\int_{0}^{h} \exp (A s) B(s) d s$. The discretized system of (22) at rate $1 / h$ is as follows

$$
x[l+1]=A_{d} x[l]+B_{d} u[l], y[l]=C x[l]
$$

Denote $\bar{b}_{i}=\int_{0}^{h_{i}} \exp (A s) b_{i} d s, i=1, \ldots, m$, where $b_{i}$ is the $i$ th column of the matrix $B$. Define

$$
\begin{aligned}
\bar{A} & =A_{d}^{N}, \quad \bar{B}=\left[\begin{array}{lll}
\bar{B}_{1} & \cdots & \bar{B}_{m}
\end{array}\right] \\
\bar{B}_{i} & =\left[\begin{array}{lll}
A_{d}^{n_{i}\left(N_{i}-1\right)} \bar{b}_{i} & \cdots & \bar{b}_{i}
\end{array}\right], \\
\bar{F}_{k} & =\left[\begin{array}{lll}
\hat{f}_{1}(k)^{T} & \cdots & \hat{f}_{m}(k)^{T}
\end{array}\right]^{T} \\
\hat{f}_{i}(k) & =\left[\begin{array}{lll}
f_{i, 0}(k)^{T} & \cdots & f_{i, N_{i}-1}(k)^{T}
\end{array}\right]^{T}
\end{aligned}
$$

for $i=1, \ldots, m$, where $\bar{N}=\sum_{i=1}^{m} N_{i}$. Define

$$
\tilde{B}(j)=\left[\begin{array}{lll}
\hat{b}_{1}(j) & \ldots & \hat{b}_{m}(j)
\end{array}\right], j=0,1, \ldots, N .
$$

where $\hat{b}_{i}(j) \in \mathcal{R}^{n \times N_{i}}, j=0, \cdots, N$ be defined as

$$
\begin{aligned}
& \hat{b}_{i}(0)=0 \\
& \hat{b}_{i}\left(v_{i}\right)=\left[\begin{array}{llll}
\check{b}_{i}\left(v_{i}\right) & 0 & \cdots & 0
\end{array}\right], \\
& \hat{b}_{i}\left(n_{i}+v_{i}\right)=\left[\begin{array}{lllll}
A_{d}^{v_{i}} \bar{b}_{i} & \breve{b}_{i}\left(v_{i}\right) & 0 & \cdots & 0
\end{array}\right], \\
& \cdots \\
& \hat{b}_{i}\left(\left(N_{i}-1\right) n_{i}+v_{i}\right)= \\
& {\left[\begin{array}{llllll}
A_{d}^{\left(N_{i}-2\right) n_{i}+v_{i}} \bar{b}_{i} & \cdots & A_{d}^{n_{i}+v_{i}} \vec{b}_{i} & A_{d}^{v_{i}} \bar{b}_{i} & \breve{b}_{i}\left(v_{i}\right)
\end{array}\right] .}
\end{aligned}
$$

where $\check{b}_{i}\left(v_{i}\right)=\int_{0}^{v_{i} h} \exp (A s) b_{i} d s, v_{i}=1, \ldots, n_{i}$. Obviously, $\check{b}_{i}\left(n_{i}\right)=\bar{b}_{i}$ and

$$
\hat{b}_{i}(N)=\left[\begin{array}{llll}
A_{d}^{\left(N_{i}-1\right) n_{i}} \bar{b}_{i} & \cdots & A_{d}^{n_{i}} \bar{b}_{i} & \bar{b}_{i}
\end{array}\right] .
$$

It is easy to find that $\bar{B}(j) \in \mathcal{R}^{m \times \bar{N}}$ and $\bar{B}(N)=\bar{B}$. From (22), we have

$$
x\left(k h_{0}+j h\right)=A_{d}^{j} x\left(k h_{0}\right)+V_{k}(j) y\left(k h_{0}\right),
$$


where $V_{k}(j)=\int_{k h_{0}}^{k h_{0}+j h} \exp \left[A\left(k h_{0}+j h-t\right)\right] B F(t) d t=$ $\sum_{i=1}^{m} V_{k}(j, i)$, and

$$
\begin{aligned}
& V_{k}(j, i) \\
= & \sum_{l=0}^{\bar{n}_{i j}-1} \int_{k h_{0}+l h_{i}}^{k h_{0}+(l+1) h_{i}} \exp \left[A\left(k h_{0}+j h-t\right)\right] b_{i} f_{i l}(k) d t \\
& +\int_{k h_{0}+\tilde{n}_{i j} h_{i}}^{k h_{0}+j h} \exp \left[A\left(k h_{0}+j h-t\right)\right] b_{i} f_{i, \bar{n}_{i j}}(k) d t,
\end{aligned}
$$

where $\bar{n}_{i j}$ is a integer satisfying $\bar{n}_{i j} n_{i} \leq j<\left(\bar{n}_{i j}+1\right) n_{i}$. Obviously $0 \leq \bar{n}_{i j} \leq N_{i}$ for all $j$. Note that $f_{i l}(k)$ is a constant vector. We can find that $V_{k}(j, i)=\hat{b}_{i}(j) \hat{f}_{i}(k)$, where $v_{i}=j-\bar{n}_{i j} n_{i}$. Hence

$$
\begin{aligned}
x\left(k h_{0}+j h\right) & =A_{d}^{j} x\left(k h_{0}\right)+\sum_{i=1}^{m} \hat{b}_{i}(j) \hat{f}_{i}(k) y\left(k h_{0}\right) \\
& =A_{d}^{j} x\left(k h_{0}\right)+\bar{B}(j) \bar{u}\left(k h_{0}\right) \\
\bar{u}\left(k h_{0}\right) & =\bar{F}_{k} y\left(k h_{0}\right),
\end{aligned}
$$

where $\bar{u}(t)$ is an augmented input and maintains constant on the interval $\left[k h_{0},(k+1) h_{0}\right)$. The discrete formula of above equations are

$$
\begin{aligned}
x[k N+j] & =A_{d}^{j} x[k N]+\bar{B}(j) \bar{u}[k N], \\
\bar{u}[k N] & =\bar{F}_{k} y(k N),
\end{aligned}
$$

where $\vec{u}[k N+j]$ will maintain constant for $j=0, \cdots, N-$ 1. It is not difficult to find that

$$
x[(k+1) N]=\bar{A} x[k N]+\bar{B} \bar{u}[k N],
$$

which is the discretized state equation of CT system (22) with sampling time $h_{0}$. With (30), we can easily compute the discretized state response with sampling time $h$.

If the multirate controller (20) is assumed to be periodic piecewise constant, the transition matrix (for period $h_{0}$ ) of the closed-loop system becomes [1] $\Phi=\bar{A}+\bar{B} \bar{F} C$, where $\bar{F}=\left[\begin{array}{lllll}f_{1,0}^{T} & \cdots & f_{1, N_{1}-1}^{T} & \cdots & f_{m, N_{m}-1}^{T}\end{array}\right]^{T}$. From (31), we can easily obtain the above transition matrix. It is shown in [1] that for almost every $h_{0}$, the matrix $\vec{B} \vec{F}$ can be assigned an arbitrary value by suitable choice of $\bar{F}$ if $(A, B)$ is controllable and if $N_{i} \geq v_{i}, i=1, \ldots, m$, where $v_{i}$ is a locally minimum controllability index of $(A, B)$ because $\bar{B}$ has rank $n$ in such case. If $(C, A)$ is observable, then the eigenvalues of $\Phi$ can be assigned arbitrarily for almost every $h_{0}$ by suitable choice of $\bar{F}$.

It is well known that a controller designed in this way and applied to a linear system will achieve the desired closed loop behavior in the output sampling instants, but is likely to result in strong oscillation between sampling instants. An effective approach to improving intersample behavior is to compute a hold function which minimizes a quadratic performance index

$$
\min _{u[k]}\left\{J=\int_{0}^{\infty}\left[x^{T}(t) Q x(t)+u^{T}(t) R \dot{u}(t)\right] d t\right\}
$$

where $R>0$ and $Q \geq 0$ are constant. The sampling of the performance index (32) at rate $1 / h$ yields the equivalent DT weights $R_{d}=\int_{0}^{h}\left(\bar{H}^{T}(t) Q \bar{H}(t)+R\right) d t, Q_{d}=$ $\int_{0}^{h}\left(e^{A t}\right)^{T} Q e^{A t} d t$, and $S_{d}=\int_{0}^{h}\left(e^{A t}\right)^{T} Q \bar{H}(t) d t$, where $\bar{H}(t)$ $\int_{0}^{t} e^{A s} B d s$. Then the sampled-data optimal control problem becomes finding a multirate feedback control law (20) to minimize the following DT performance index

$$
J=\sum_{j=0}^{\infty}\left[\begin{array}{ll}
x^{T}[l] & u^{T}[l]
\end{array}\right]\left[\begin{array}{ll}
Q_{d} & S_{d} \\
S_{d}^{T} & R_{d}
\end{array}\right]\left[\begin{array}{l}
x[l] \\
u[l]
\end{array}\right]
$$

with the piecewise COF $u[l]$ at rate $1 / h$. We can rewrite (31) as the following LTI system

$$
\begin{aligned}
x_{N}[k+1] & =\bar{A} x_{N}[k]+\bar{B} u_{N}[k], \\
y_{N}[k] & =C x_{N}[k], \\
u_{N}[k] & =\bar{F}_{k} y_{N}[k],
\end{aligned}
$$

which can be seen as the sampling of system (23) at $N$, where $x_{N}[k]=x[k N], y_{N}[k]=y[k N], u_{N}[k]=$ $\bar{u}[k N], k \geq 0$, with initial condition $x_{N}[0]=x[0]$. The input $u[k N+j]$ in (23) and (33) can be written as

$$
u[k N+j]=F_{j}(k) y[k N]=W_{j+1} u_{N}[k],
$$

for $j=0, \ldots, N-1$, where $W_{j}=\left\{w_{i l}^{j}\right\} \in \mathcal{R}^{m \times \bar{N}}, j=$ $1, \ldots, N$, are defined as

$$
w_{i l}^{j}=\left\{\begin{array}{lll}
1, & l=\sum_{r=1}^{i-1} N_{r}+\bar{n}_{i j}+1 & , \quad i=1, \ldots, m \\
0, & \text { Otherwise } & l=1, \ldots, \bar{N}
\end{array}\right.
$$

The time-invariant system (34) can be seen as a statesampled representation of system (23), feeding by an augmented input vector (36) and producing an augmented output vector (35). It is easy to find that system (34) is stable if and only if system (23) is asymptotically stable.

So, with initial condition $x_{N}[0]=x[0],(33)$ can be rewritten as

$$
J=\sum_{k=0}^{\infty}\left[\begin{array}{ll}
x_{N}^{T}[k] . & u_{N}^{T}[k]
\end{array}\right]\left[\begin{array}{ll}
\tilde{Q} & \tilde{S} \\
\tilde{S}^{T} & \tilde{R}
\end{array}\right]\left[\begin{array}{l}
x_{N}[k] \\
u_{N}[k]
\end{array}\right],
$$

where $\tilde{Q}, \tilde{S}$ and $\tilde{R}$ are time-invariant matrices

$$
\begin{aligned}
\tilde{Q}= & \sum_{j=0}^{N-1}\left(A_{d}^{j}\right)^{T} Q_{d} A_{d}^{j} \\
\tilde{R}= & \sum_{j=0}^{N-1} W_{j+1}^{T} R_{d} W_{j+1}+\sum_{j=1}^{N-1}\left(\bar{B}(j)^{T} Q_{d} \bar{B}(j)\right. \\
& \left.+\bar{B}(j)^{T} S_{d} W_{j+1}+W_{j+1}^{T} S_{d}^{T} \bar{B}(j)\right) \\
\tilde{S}= & \sum_{j=0}^{N-1}\left(A_{d}^{j}\right)^{T} S_{d} W_{j+1}+\sum_{j=1}^{N-1}\left(A_{d}^{j}\right)^{T} Q_{d} \bar{B}(j) .
\end{aligned}
$$

Theorem 3 Assume the CT system (22) is given. The optimal design of piecewise output feedback control law (20) minimizing (32) can be reduced to that of a COF control law $\bar{F}$ minimizing (39). 
The discrete time-invariant system (34) has the state $x_{N}[k]=x[k N]$. From (37), any output feedback control law $u_{N}[k]=\bar{F} x_{N}[k]$ can be written as $u[k N+j]=$ $W_{j+1} \bar{F} x[k N], \quad j=0, \ldots, N-1$, where $W_{j}$ are defined as in (38).

The above derivation for single plant can be easily extended to simultaneous $\mathrm{LQ}$ optimal design for $r \mathrm{CT}$ systems (17) by the same piecewise COF controller (20). From last subsection, the CT system model (17) and the periodic piecewise COF control law (20) can be reduced to the following DT system models

$$
\begin{aligned}
x_{i, N}[k+1] & =\bar{A}_{i} x_{i, N}[k]+\bar{B}_{i} u_{i, N}[k], \\
y_{i, N}[k] & =C_{i} x_{i, N}[k], \\
u_{i, N}[k] & =\bar{F} y_{i, N}[k],
\end{aligned}
$$

where $\bar{A}_{i}, \bar{B}_{i}$ is defined by (24) and (25), respectively.

Theorem 4 Assume that the $\mathrm{CT}$ systems (17) are given. The simultaneous optimal design of piecewise output feedback control law (20) minimizing (18) can be reduced to that of a COF control law $\bar{F}$ minimizing

$$
J_{i}=\sum_{k=0}^{\infty}\left[\begin{array}{ll}
x_{i, N}^{T}[k] & u_{i, N}^{T}[k]
\end{array}\right]\left[\begin{array}{cc}
\tilde{Q}_{i} & \tilde{S}_{i} \\
\tilde{S}_{i}^{T} & \tilde{R}_{i}
\end{array}\right]\left[\begin{array}{l}
x_{i, N}[k] \\
u_{i, N}[k]
\end{array}\right]
$$

for the LTI systems $(43,44)$, where $\tilde{Q}_{i}, \tilde{R}_{i}$ and $\tilde{S}_{i}$ can be determined as in (40), (41) and (42) respectively for $i=1, \ldots, r$.

Based on the above theorem, after selecting the frame period $h_{0}$ and the input multiplicities $N_{i}$, Algorithm 1 of Section 3 can be used to solve the feedback gain $\vec{F}$.

\section{Examples}

Consider two plants

$$
G_{1}(s)=\frac{1}{s+1}, \quad G_{2}(s)=\frac{a s+1}{(s+1)(s-1)},
$$

where $a$ is a real constant with $a \neq 1$. It is not difficult to find when $a<-1$, they are not simultaneously stabilizable by an LTI controller. Now we use the presented approach to find a simultaneous stabilizing periodic controller for $a=-2$. Let the sampling time be $\tau=1$. When $N=1$, the ILMI algorithm cannot obtain a feasible solution, but when we select $N=2$, after 25 iterations we get the following result

$$
\begin{gathered}
\bar{F}=\left[\begin{array}{lll}
7.493 & -4.484
\end{array}\right], P_{1}=\left[\begin{array}{cc}
45.6598 & -0.0004 \\
-0.0004 & 0.0010
\end{array}\right], \\
P_{2}=\left[\begin{array}{ccc}
720.1515 & -668.5406 & -0.3663 \\
-668.5406 & 657.4452 & 0.3294 \\
-0.3663 & 0.3294 & 0.0922
\end{array}\right] .
\end{gathered}
$$

This means that the above two plants can be simultaneously stabilizable by a 2-periodic piecewise COF gain.

\section{Conclusion}

In this paper, simultaneous piecewise output feedback LQ optimal control is addressed using ILMI approach for multiple LTI CT systems. It is shown that the design of the simultaneous LQ optimal feedback gain can be reduced to a set of coupled matrix inequalities. An iterative LMI algorithm is proposed to solve the feasibility. From numerical experience, the algorithm leads to a feasible solution of the simultaneous LQ optimal control problem whenever a controller exists. However, even if a simultaneous stabilizing controller exists, it remains to be established that the ILMI algorithm would always give a feasible solution.

\section{References}

[1] M. Araki and T. Hagiwara. Pole assignment by multirate sampled-data output feedback. Int. J. Control, 44:1661-1673, 1986.

[2] M. Araki, T. Hagiwara, and H. Somak. Application of multilevel multirate sampled-data controllers to simultaneous pole assignment problem. In Proc. 31 st IEEE Conf. Dec. Control, pages 1762-1767, 1992.

[3] Y.-Y. Cao and Y.-X. Sun. Static output feedback simultaneous stabilization: ILMI approach. Int. $J$. Control, 70(5):803-814, 1998.

[4] Y.-Y. Cao, Y.-X. Sun, and J. Lam. Simultaneous stabilization via static output feedback and state feedback. IEEE Trans. Automat. Control, 44(6):1277-1282, 1999.

[5] A.B. Chammas and C.T. Leondes. Pole assignment by piecewise constant output feedback. Int. J. Control, 29(1):31-38, 1979.

[6] P. Colaneri, V. Kucera, and C.E. de Souza. Output stabilization for periodic systems. In Proc. 1998 American Control Conf., pages 2795-2796, Philadelphia, USA, 1998.

[7] P.T. Kabamba. Control of linear systems using generalized sampled-data hold functions. IEEE Trans. Automat. Control, 32:772-783, 1987.

[8] P. P. Khargonekar, K. Poolla, and A. Tannenbaum. Robust control of linear time invariant plants using periodic compensation. IEEE Trans. Automat. Control, 30:1088-1096, 1985.

[9] V. Kučera and C. de Souza. A necessary and sufficient condition for output feedback stabilizability. Automatica, 31(9):1357-1359, 1995.

[10] J. Lam and Y.-Y. Cao. Simultaneous linearquadratic optimal control design via static output feedback. Int. J. of Robust and Nonlinear Control, 9(9):551-558, 1999. 